\title{
Characterization of biodegradable polymers with capacitive field-effect sensors
}

\author{
S. Schusser ${ }^{\mathrm{a}, \mathrm{b}}$, A. Poghossian ${ }^{\mathrm{a}, \mathrm{b}, *}$, M. Bäcker ${ }^{\mathrm{a}, \mathrm{b}}$, M. Leinhos ${ }^{\mathrm{a}, \mathrm{b}}$, P. Wagner $^{\mathrm{c}}$, M.J. Schöning $^{\mathrm{a}, \mathrm{b}}$ \\ a Institute of Nano- and Biotechnologies (INB), Aachen University of Applied Sciences, Campus Jülich, 52428 Jülich, Germany \\ b Peter Grünberg Institute (PGI-8), Forschungszentrum Jülich GmbH, 52525 Jülich, Germany \\ ' Institute for Materials Research (IMO), Hasselt University, 3590 Diepenbeek, Belgium
}

\section{A R T I C L E I N F O}

\section{Article history:}

Available online 16 August 2012

\section{Keywords:}

Field-effect sensor

(Bio)degradation

Poly(D,L-lacticacid)

Real-time monitoring

$C-V$ method

Impedance spectroscopy

\begin{abstract}
A B S T R A C T
In vitro studies of the degradation kinetic of biopolymers are essential for the design and optimization of implantable biomedical devices. In the presented work, a field-effect capacitive sensor has been applied for the real-time and in situ monitoring of degradation processes of biopolymers for the first time. The polymer-covered field-effect sensor is, in principle, capable to detect any changes in bulk, surface and interface properties of the polymer induced by degradation processes. The feasibility of this approach has been experimentally proven by using the commercially available biomedical polymer poly(D,L-lactic acid) (PDLLA) as a model system. PDLLA films of different thicknesses were deposited on the $\mathrm{Ta}_{2} \mathrm{O}_{5}{ }^{-}$ gate surface of the field-effect structure from a polymer solution by means of spin-coating method. The polymer-modified field-effect sensors have been characterized by means of capacitance-voltage and impedance-spectroscopy method. The degradation of the PDLLA was accelerated by changing the degradation medium from neutral $(\mathrm{pH} 7.2)$ to alkaline $(\mathrm{pH} 9)$ condition, resulting in drastic changes in the capacitance and impedance spectra of the polymer-modified field-effect sensor.
\end{abstract}

(c) 2012 Elsevier B.V. All rights reserved.

\section{Introduction}

Although biomaterials have already made an enormous impact in biomedical research and clinical practice (e.g., in drugdelivery systems, as scaffolds for tissue engineering, or orthopedic implants), there is a need for new biodegradable synthetic polymers and implantable devices with controlled/predicted biodegradability [1-6]. The mechanisms of polymer degradation in vivo are complex, because of the multiple active components in cells and the extracellular fluid. Generally, hydrolysis is the most probable degradation mechanism for polymers in vivo [7]. Synthetic biodegradable polymers contain hydrolyzable linkages along the polymer chain, which are susceptible to biodegradation by microorganisms and hydrolytic enzymes. In general, the factors affecting biodegradation include many physico-chemical parameters of the polymer such as the polymer structure and morphology (amorphous/crystalline), molecular weight, hydrophilicity/hydrophobicity, mechanical properties, etc. (see e.g., [6-10]). In addition, the degradation environment (e.g., presence or absence of oxygen, microorganisms, enzymes, $\mathrm{pH}$, ionic strength, temperature) strongly influences the biodegradability and kinetics of biodegradation processes. For any application,

\footnotetext{
* Corresponding author at: Institute of Nano- and Biotechnologies (INB), Aachen University of Applied Sciences, Campus Jülich, 52428 Jülich, Germany.

E-mail address: a.poghossian@fz-juelich.de (A. Poghossian).
}

the in vitro study of the degradation kinetics of the biopolymers is essential not only for a fundamental understanding of the nature of the degradation process but also for the design and optimization of implantable biomedical devices.

Common techniques used to quantify degradation (e.g., determination of mass loss, shortening of the chain length or shift of the glass-transition temperature towards lower values) are not suitable for real-time measurements because of their destructive manner and thus, limit studies on degradation kinetics in respect to throughput and precision [5]. In this context, analyzing techniques capable for real-time in situ monitoring of the degradation kinetics of polymers are therefore highly appreciated. Recently, several approaches and transducer principles have been suggested for monitoring the dissolution or enzymatic degradation of those polymer films. These include, for instance, surface plasmon resonance, quartz crystal microbalance, electrochemical impedance spectroscopy (IS) or scanning photo-induced IS [11-14].

In this report, we present experimental results on polymer degradation investigated by means of capacitive field-effect electrolyte-insulator-semiconductor (EIS) sensors, as a novel and very promising platform for the real-time in situ monitoring of the degradation kinetics of biopolymers. The commercially available biodegradable polymer poly(lactic acid) (PLA) was chosen as a model system, because of its exceptional importance, biocompatibility and its use in numerous approved medical devices and pharmaceutical applications. 


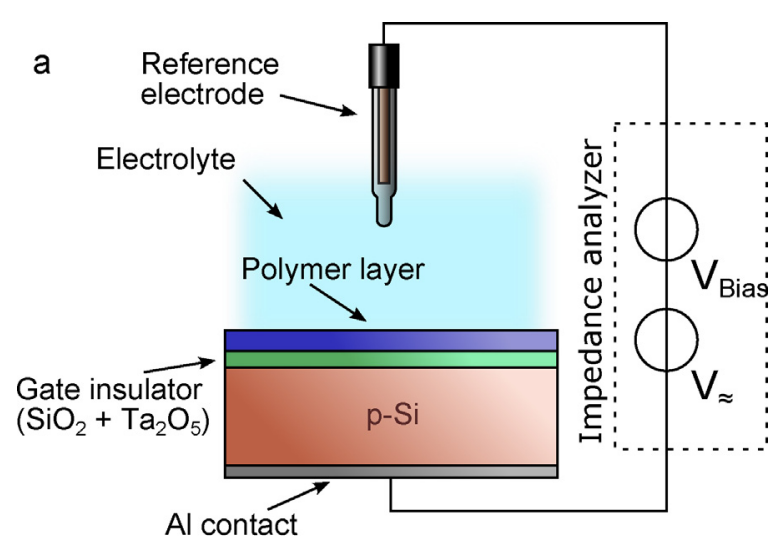

b

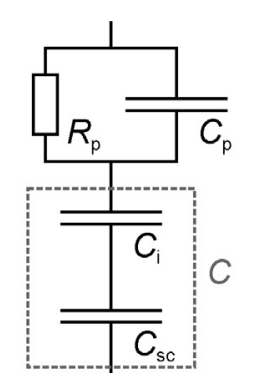

Fig. 1. Schematic of the measurement set-up and layer structure of a field-effect PMEIS sensor (a) and its simplified equivalent circuit (b) comprising the effective capacitance of the EIS sensor $C ; C$ is consisting of the insulator capacitance $C_{\mathrm{i}}$ and the capacitance of the space-charge region $C_{\mathrm{sc}}$. The polymer layer is defined by and the geometric resistance $R_{\mathrm{p}}$ and the capacitance $C_{\mathrm{p}}$.

\section{Sensor-functioning principle}

Fig. 1 shows a schematic of the layer structure, measuring setup (a) and a simplified equivalent circuit (b) of a polymer-modified EIS sensor (further referred to as PMEIS). The sensor structure consists of a silicon substrate covered with a gate insulator (in this study, $\mathrm{SiO}_{2}-\mathrm{Ta}_{2} \mathrm{O}_{5}$ ) and an ohmic contact on the backside. A thin layer of the polymer is deposited on the gate insulator and contacted to the degradation medium. In previous experiments, field-effect sensors have been widely utilized for the detection of various (bio-)chemical quantities, like $\mathrm{pH}$, ion- and analyte concentration, charged macromolecules as well as cellular signals (see e.g., [15-23]). The field-effect sensor is, in principle, capable to detect any changes in the bulk, surface and interface properties of the polymer (thickness, coverage, dielectric constant, surface potential, local pH at or nearby the polymer surface, etc.) induced by the degradation processes.

For the PMEIS structure, the polymer layer can be described as a parallel network of the polymer geometric resistance, $R_{\mathrm{p}}$, and the capacitance, $C_{\mathrm{p}}$, which is in series with the bare insulator-semiconductor structure [24,25]. The experimentally measured capacitance of the PMEIS structure, $C_{\text {meas }}$, can be expressed as (the electrochemical double-layer capacitance at the polymer/electrolyte interface is assumed to be much greater than other capacities in the system and can thus be neglected) [26]:

$$
C_{\text {meas }}=C \frac{1+R_{\mathrm{p}}^{2} C_{\mathrm{p}}^{2} \omega^{2}}{1+R_{\mathrm{p}}^{2}\left(C C_{\mathrm{p}}+C_{\mathrm{p}}^{2}\right) \omega^{2}}
$$

with

$C=\frac{C_{\mathrm{i}} C_{\mathrm{sc}}}{C_{\mathrm{i}}+C_{\mathrm{sc}}}$

where $C$ is the capacitance of the original EIS structure without the polymer layer, $C_{\mathrm{i}}$ is the gate-insulator capacitance, $\omega=2 \pi f, f$ is the measuring frequency and $C_{\mathrm{sc}}$ is the space-charge capacitance of the Si, which is among others a function of the gate voltage applied to the PMEIS structure and interfacial potential at the polymer/electrolyte and polymer/gate-insulator interface, respectively. Thus, any changes in the polymer resistance/capacitance as well as in interfacial potentials induced by the polymer degradation will modulate the global capacitance/impedance of the PMEIS structure that can be used as an indicator of the polymer degradation. The degradation rate can be evaluated from the time-dependent capacitance-voltage $(C-V)$ or IS characteristics of the PMEIS structure.

\section{Experimental}

\subsection{Fabrication of EIS sensors and polymer deposition}

For the degradation experiments, the EIS chips (with a chip size of $10 \mathrm{~mm} \times 10 \mathrm{~mm}$ ) consisting of an $\mathrm{Al}-\mathrm{p}-\mathrm{Si}-\mathrm{SiO}_{2}-\mathrm{Ta}_{2} \mathrm{O}_{5}$ structure $(\mathrm{p}-\mathrm{Si}, \rho=5-10 \mathrm{~cm}), 300 \mathrm{~nm} \mathrm{Al}$ as rear-side contact layer, $30 \mathrm{~nm} \mathrm{SiO}{ }_{2}$ and $60 \mathrm{~nm} \mathrm{Ta}_{2} \mathrm{O}_{5}$ were fabricated. Then, the chips were covered with a polymer layer of PLA. PLA is one of the most important and best characterized degradable polymers for medical applications and attracted a high interest due to its biocompatible, chemical structure and its decomposition products that can be completely metabolized in vivo $[27,28]$.

In this study, the poly(D,L-lactic acid) (PDLLA) benchmark biopolymer RESOMER ${ }^{\circledR}$ R 202 H (Evonik Röhm GmbH, Germany) was chosen as a model biopolymer for the degradation measurements. RESOMER ${ }^{\circledR}$ R $202 \mathrm{H}$ is designed for controlled release applications and consists of a 50:50 ratio of $\mathrm{D} / \mathrm{L}$-lactic acid with a molecular weight in the range of $M_{w}=10,000-18,000$. Fig. 2 depicts the chemical structure of this isotactic polymer and a simplified scheme of the hydrolytic degradation process. A more detailed description of this process can be found elsewhere [29].

PDLLA films of different thicknesses were deposited on the $\mathrm{Ta}_{2} \mathrm{O}_{5}$ surface from polymer solutions by means of a spin-coating method. The desired range of the layer thickness has been achieved by dissolving varying concentrations of the polymer in acetone (see Fig. 3). The thickness of the polymer layer was determined by profilometry. For the degradation experiments, sensors were spin-coated with a polymer solution of $100 \mathrm{mg} / \mathrm{mL}$ concentration, resulting in a PDLLA layer of approximately $500 \mathrm{~nm}$ thickness.

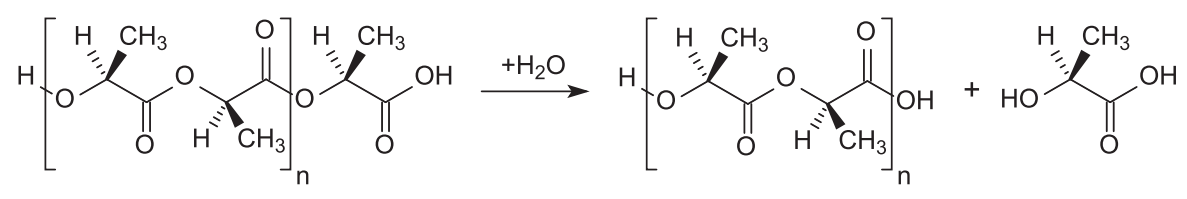

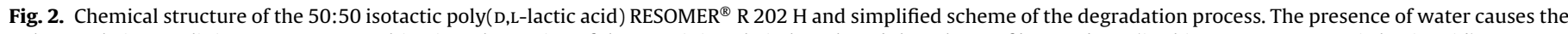
polymer chain to split into two parts resulting in a shortening of the remaining chain length and the release of by-products (in this case, a monomeric lactic acid). 


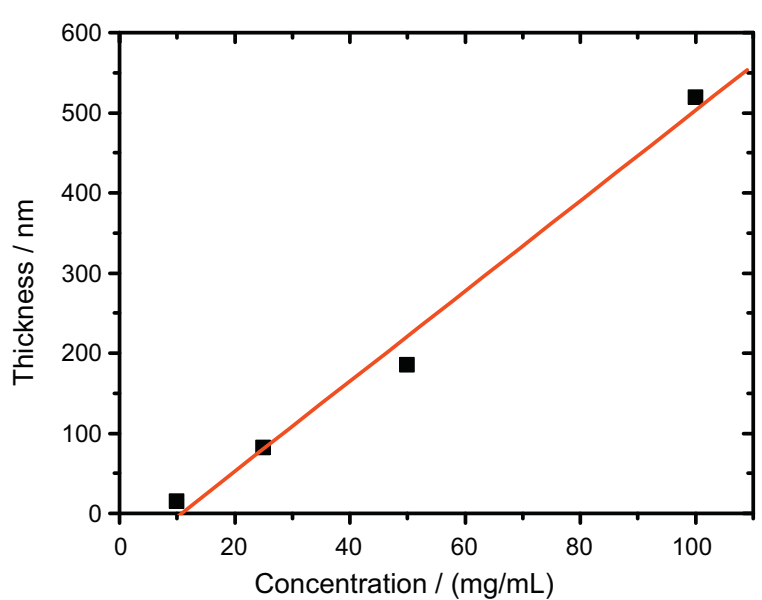

Fig. 3. Concentration-dependent thickness of spin-coated RESOMER ${ }^{\circledR}$ R $202 \mathrm{H}$ solved in acetone and investigated by means of profilometry.

\subsection{Measurement set-up}

The sensor chip was mounted into a home-made measuring cell, sealed by an O-ring, and contacted to the degradation solution on its front side. The contact area of the polymer with the solution was about $0.5 \mathrm{~cm}^{2}$. The PMEIS structures were characterized before and during polymer degradation by means of $C-V$ and IS method using an impedance analyzer (IM6, Zahner Elektrik, Germany). For comparison, the $C-V$ characteristics of a bare EIS sensor without polymer layer were also recorded. During the measurements, a DC polarization voltage is applied between a conventional liquid-junction $\mathrm{Ag} / \mathrm{AgCl}$ reference electrode (Metrohm) and the rear-side Al contact (see Fig. 1). A small AC voltage $(20 \mathrm{mV})$ is superimposed to the system in order to measure the complex impedance of the PMEIS structure. The $C-V$ curves were periodically captured at a frequency of $158.5 \mathrm{~Hz}$. IS characteristics were recorded in the accumulation range of the $C-V$ curve (at an applied gate voltage of $-2 \mathrm{~V}$ ) in the frequency range from $0.1 \mathrm{~Hz}$ to $1 \mathrm{MHz}$. The degradation experiments were carried out at $37^{\circ} \mathrm{C}$. For data processing, a special program based on LabVIEW (National Instruments) was developed. This program is capable to run time-controlled $C-V$ and IS measurements.

\section{Results and discussion}

Fig. 4 represents an example of real-time monitoring of polymer degradation with a field-effect structure. In this experiment, the $C-V$ characteristic of a bare EIS (a) and the time-dependent evolution (over a time period of about seven days) of the $C-V$ characteristic of a PMEIS sensor with a $500 \mathrm{~nm}$ thick PDLLA layer was recorded. The $C-V$ characteristic of the bare EIS sensor exhibits the distinctive characteristics of an ideal EIS structure that can be assigned to three operation modes ( $\mathrm{Si}$ substrate is p-doped) [30]:

- Accumulation: The electrolyte is negatively biased with respect to the Si bulk resulting in an accumulation of the positively charged holes (majority carriers) at the silicon-insulator interface. In this mode, the capacitance of the insulator prevails setting the capacitance to a steady maximum of approximately $52 \mathrm{nF}$.

- Depletion: At slightly negative values of the potential of the electrolyte with respect to the Si bulk, the charge carriers (holes) are repelled from the silicon-insulator interface forming a depletion
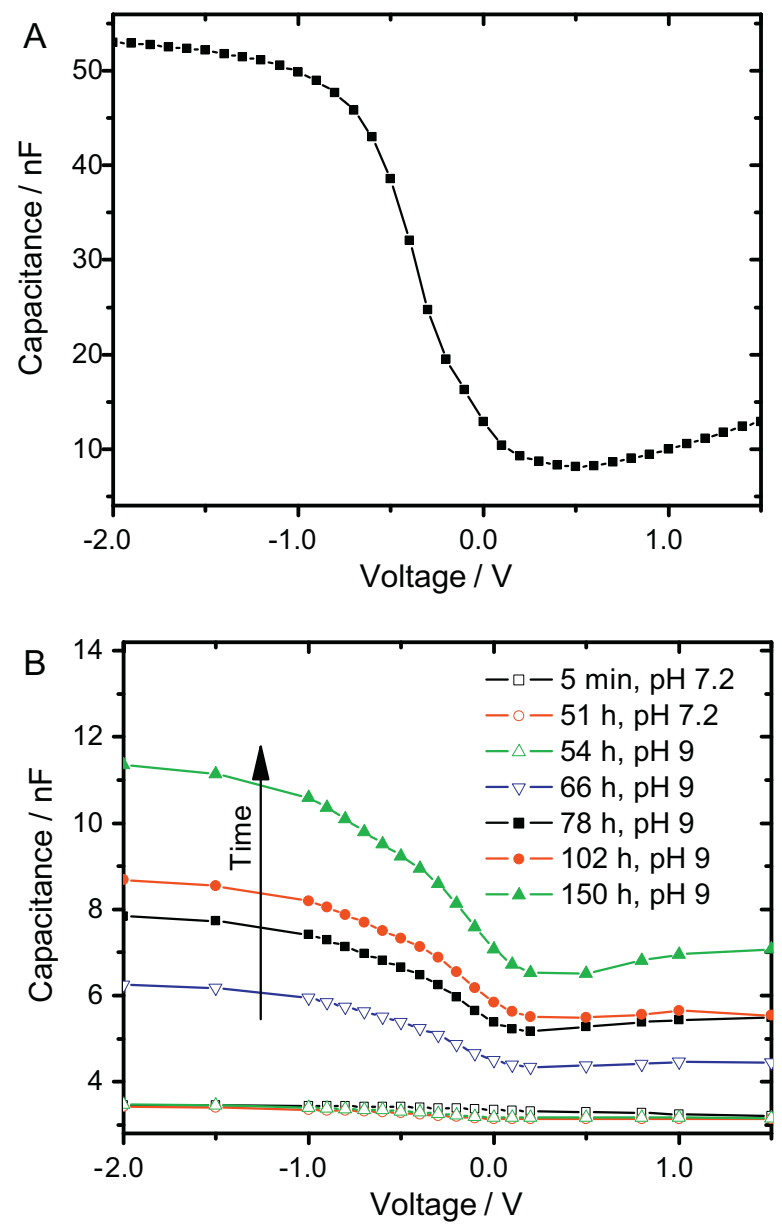

Fig. 4. $C-V$ characteristic of the bare EIS sensor structure (A) and time-dependent curves of the PMEIS sensor during degradation (B). Even after 2 days of exposure to $\mathrm{pH} 7.2$ solution, the $C-V$ characteristic remains at the initial level of about $3.5 \mathrm{nF}$. After changing the degradation medium to $\mathrm{pH} 9$, the capacitance value increases and the typical shape of the $C-V$ characteristic appears.

zone that acts as an additional capacitance in series with the insulating layer resulting in a decline of the total capacitance.

- Inversion: A further increase of the potential (electrolyte is strongly positively biased with respect to the Si) leads to an inversion of the charge carriers at the silicon-insulator interface. For an ideal EIS structure, a further increase of the potential affects the size of the space-charge region imperceptibly settling the total capacitance to a minimum (high-frequency case). The present device shows a slight, linear increase at this region associated to minor imperfections of the insulating layer.

It is known, that PDLLA has a slow degradability in neutral $\mathrm{pH}$ solutions and shows a higher degradability in alkaline solutions [6]. Therefore, during the first $53.5 \mathrm{~h}$ of monitoring, the polymer layer was exposed to a phosphate-buffered medium of $\mathrm{pH} 7.2$, that mimics physiological conditions. During this time period, practically no changes in the capacitance have been observed, revealing that no (or negligible) degradation was occurred (see Fig. 4(b)). This is in good agreement with previous studies [6]. As it could be expected, in comparison with a $C-V$ curve of the bare EIS structure, the $C-V$ curve of the PMEIS sensor is strongly deformed yielding to an unusual and practically flat curve shape. The maximum capacitance in the accumulation range amounted to the $3.5 \mathrm{nF}$ and was mainly determined by the capacitance of the polymer layer.

In order to induce a faster polymer degradation, the PDLLA layer was further exposed to an alkaline buffer solution of $\mathrm{pH} 9$, resulting 


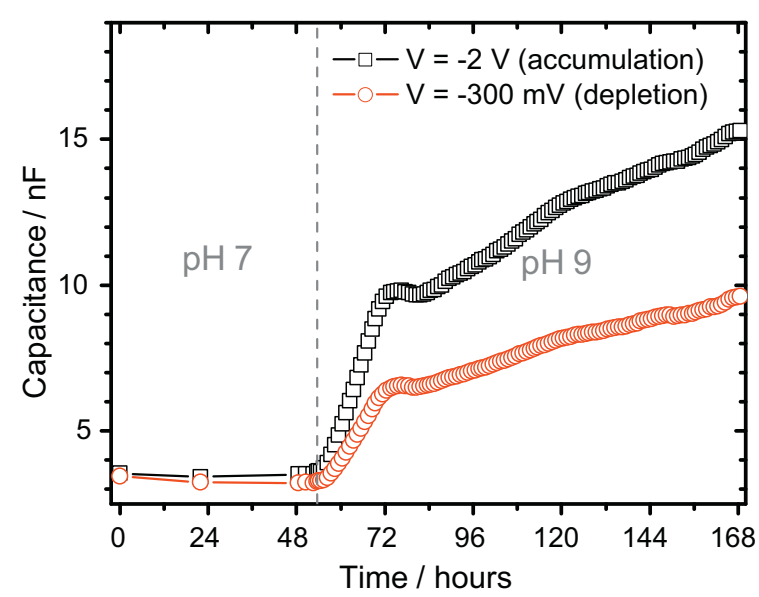

Fig. 5. Capacitance over time of the PMEIS sensor in accumulation $(V=-2 \mathrm{~V})$ and depletion $(V=-300 \mathrm{mV})$ mode. The degradation of PDLLA was accelerated by changing of the degradation medium to $\mathrm{pH} 9$ at $53.5 \mathrm{~h}$ (dashed vertical line).

in drastic changes in the capacitance of the PMEIS structure over time. Moreover, the $C-V$ curves of the PMEIS structure exhibit a usual high-frequency behavior with typical accumulation, depletion and inversion regions. The time-dependent shifts of the $C-V$ curve can be used as an indicator of polymer degradation. The degradation kinetics of the PDLLA film can clearly be seen in Fig. 5, where the capacitance changes over time in accumulation (at gate voltage of $V_{\mathrm{G}}=-2 \mathrm{~V}$ ) and depletion (at $V_{\mathrm{G}}=-0.3 \mathrm{~V}$ ) regions (evaluated from the $C-V$ curves in Fig. 4) are presented. Even after seven days of exposure of the PDLLA film to the alkaline medium, the capacitance of the PMEIS sensor was smaller than that of a bare EIS sensor, indicating that the polymer degradation is not yet completed. These results are supported by the optical microscopy image of the sensor surface after degradation (Fig. 6). As a result of the degradation process, the sensor surface is still partially covered with the polymer film having large pores and thinned regions.

Similar results can be extracted from the impedance spectra (see Fig. 7). Starting at a mostly capacitive behavior between $0.1 \mathrm{~Hz}$ and $1 \mathrm{kHz}$, the impedance decreases and a resistive portion became visible from $10 \mathrm{~Hz}$ to $100 \mathrm{~Hz}$ during degradation. This behavior can be attributed to a process of water uptake prior to degradation, since PDLLA is predominantly eroded via bulk degradation [6]. The plateau at $10 \mathrm{kHz}$ and the subsequent decline refers to the resistance of the reference electrode and parasitic capacitance of the system, respectively.

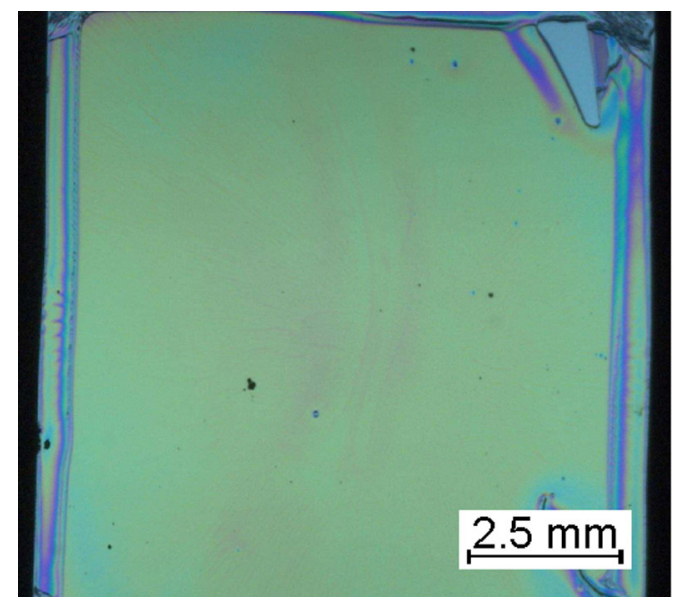

Fig. 6. Microscopic image of the surface of the PMEIS sensor with fabricated PDLLA layer before (left) and after (right) 7 days of exposure to degradation medium.

Fig. 7. Time-dependent impedance spectra of the PMEIS sensor exposed to $\mathrm{pH} 9$ solution.

For a quantitative analysis of the temporal evolution of the impedance spectra, the equivalent circuit of the PMEIS structure (see Fig. 1(b)) was fitted to the measured data. The model was expanded by a resistance $R_{\text {Ref }}$ in series and a capacitance $C_{\text {sys }}$ parallel to the PMEIS and $R_{\text {Ref }}$, representing the reference electrode and the parasitic capacitance of the system, respectively (Fig. 8(a)). The results of the fit for $C_{\mathrm{p}}$ and $R_{\mathrm{p}}$ are shown in Fig. 8(b) for fixed values of $C=50 \mathrm{nF}, C_{\text {sys }}=0.25 \mathrm{nF}$ and $R_{\mathrm{Ref}}=15 \mathrm{k} \Omega$. These results lead to the presumption that the degradation of the polymer layer proceeds in two steps:

1 After the degradation medium was changed to $\mathrm{pH}$ 9, the resistive part of the polymer layer decreased in the order of two magnitudes within the first $24 \mathrm{~h}$.

2 Afterwards, it decreased further but exhibits a slower rate indicating ongoing degradation. At the same time, the capacitance showed a change of tendency at this point from an increasing to a temporary period of a decreasing value and again followed by a slow increase.

This progress can indicate successively ongoing or partly overlapping degradation processes (namely water uptake, swelling, chemical degradation, onsetting erosion or recrystallization), which are attendant mechanisms of the degradation process that mainly define the degradation kinetics [31].

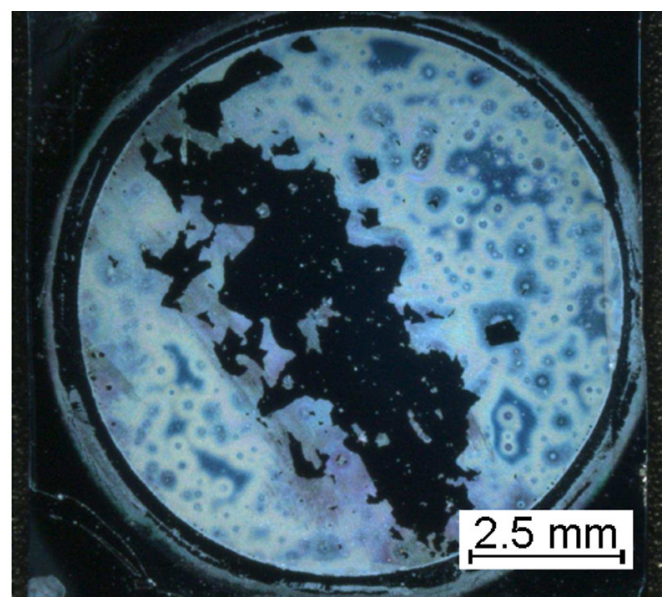


a

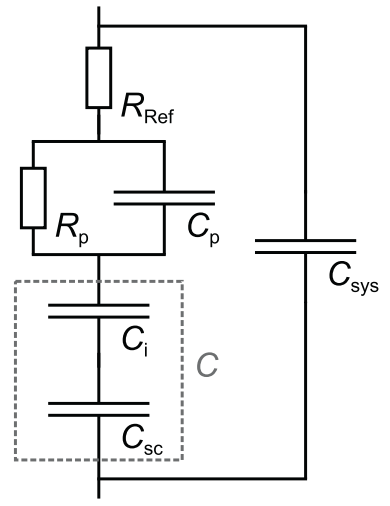

b

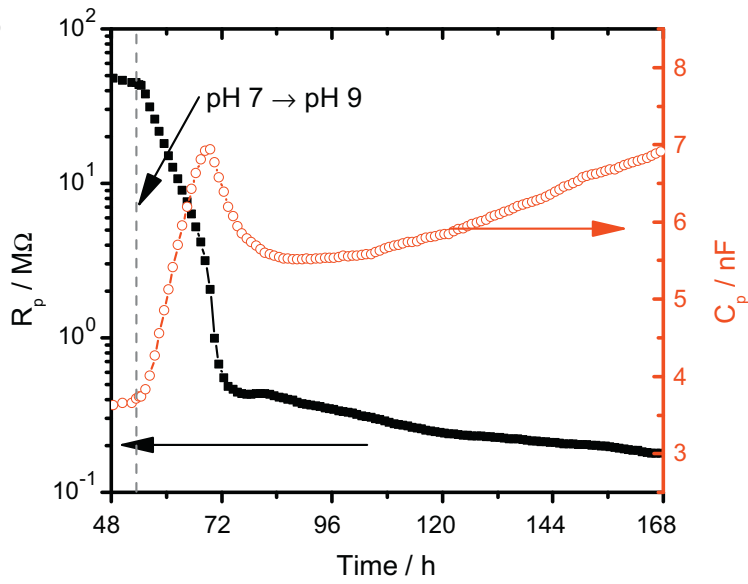

Fig. 8. Results of the time-resolved fit of the PDLLA degradation. The polymer was modeled by a parallel network of $R_{\mathrm{p}}$ and $C_{\mathrm{p}}$ as described in Section 2 .

\section{Conclusions}

Investigation and examination of the degradation process in polymers with respect to their rate and type has been often a laborious procedure, so far. By means of an EIS sensor, a system for real-time and in situ tracing of degradation processes of biopolymers has been shown. Exemplarily, the sensor signal of the accelerated degradation of PDLLA due to an alkaline treatment was tracked by $C-V$ and IS method. A simplified equivalent circuit was employed to achieve a quantitative description of the time-dependent impedance spectra. The obtained results suggest a multi-step degradation process probably due to attendant mechanisms of the degradation process, such as water uptake. These preliminary successful experiments demonstrate the potential of field-effect devices as a novel and promising tool for the real-time and in situ monitoring of polymer degradation. Subject to further studies is the formulation of a more detailed model of the PMEIS sensor for a precise description of the degradation process.

\section{Acknowledgements}

This particular study was financed through generous contributions of the European Union (through Interreg IV-A) and the government of North Rhine-Westphalia (Germany) in the frame of BioMIMedics.

\section{References}

[1] L.S. Nair, C.T. Laurencin, Biodegradable polymers as biomaterials, Progress in Polymer Science 32 (2007) 762-798.
[2] M. Martina, D.W. Hutmacher, Biodegradable polymers applied in tissue engineering research: a review, Polymer International 56 (2007) 145-157.

[3] C.-S. Ha, J.A. Gardella Jr., Surface chemistry of biodegradable polymers for drug delivery systems, Chemical Reviews 105 (2005) 4205-4232.

[4] J. Park, M. Ye, K. Park, Biodegradable polymers for microencapsulation of drugs, Molecules 10 (2005) 146-161.

[5] W. Amass, A. Amass, B. Tighe, A review of biodegradable polymers: uses, current developments in the synthesis and characterization of biodegradable polyesters, blends of biodegradable polymers and recent advances in biodegradation studies, Polymer International 47 (1998) 89-144.

[6] F. von Burkersroda, L. Schedl, A. Göpferich, Why degradable polymers undergo surface erosion or bulk erosion, Biomaterials 23 (2002) 4221-4231.

[7] D.F. Williams, S.P. Zhong, Biodeterioration/biodegradation of polymeric medical devices in situ, International Biodeterioration and Biodegradation 34 (1994) 95-130.

[8] J.-D. Gu, Microbiological deterioration and degradation of synthetic polymeric materials: recent research advances, International Biodeterioration and Biodegradation 52 (2003) 69-91.

[9] N. Lucas, C. Bienaime, C. Belloy, M. Queneudec, F. Silvestre, J.-E. Nava-Saucedo Polymer biodegradation: mechanisms and estimation techniques, Chemosphere 73 (2008) 429-442.

[10] P. Gunatillake, R. Mayadunne, R. Adhikari, Recent developments in biodegradable synthetic polymers, Biotechnology Annual Review 12 (2006) 301-347.

[11] C.J. McNeil, D. Athey, M. Ball, W.O. Ho, S. Krause, R.D. Armstrong, J. Des Wright, K. Rawson, Electrochemical sensors based on impedance measurement of enzyme-catalyzed polymer dissolution: theory and applications, Analytical Chemistry 67 (1995) 3928-3935.

[12] A. Sabot, S. Krause, Simultaneous quartz crystal microbalance impedance and electrochemical impedance measurements. Investigation into the degradation of thin polymer films, Analytical Chemistry 74 (2002) 3304-3311.

[13] S. Krause, W. Moritz, H. Talabani, M. Xu, A. Sabot, G. Ensell, Scanning photo-induced impedance microscopy - resolution studies and polymer characterization, Electrochimica Acta 51 (2006) 1423-1430.

[14] Y. Zhou, S. Jiang, S. Krause, J.-N. Chazalviel, Biosensor arrays based on the degradation of thin polymer films interrogated by scanning photoinduced impedance microscopy, Analytical Chemistry 79 (2007) 8974-8978.

[15] M.J. Schöning, N. Näther, V. Auger, A. Poghossian, M. Koudelka-Hep, Miniaturised flow-through cell with integrated capacitive EIS sensor fabricated at wafer level using Si and SU-8 technologies, Sensors and Actuators B 108 (2005) 986-992.

[16] Y. Mourzina, T. Mai, A. Poghossian, Y. Ermelenko, T. Yoshinobu, Y. Vlasov, H. Iwasaki, M.J. Schöning, $\mathrm{K}^{+}$-selective field-effect sensors as transducers for bioelectronic applications, Electrochimica Acta 48 (2003) 3333-3339.

[17] T. Yoshinobu, H. Ecken, A. Poghossian, A. Simonis, H. Iwasaki, H. Lüth, M.J Schöning, Constant-current-mode LAPS (CLAPS) for the detection of penicillin, electroanalysis 13 (2001) 733-736.

[18] A. Poghossian, M. Thust, M.J. Schöning, M. Müller-Veggian, P. Kordos, H. Lüth, Cross-sensitivity of a capacitive penicillin sensor combined with a diffusion barrier, Sensors and Actuators B 68 (2000) 260-265.

[19] C.-S. Lee, S.K. Kim, M. Kim, Ion-sensitive field-effect transistor for biological sensing, Sensors 9 (2009) 7111-7131.

[20] J. Gun, M.J. Schöning, M.H. Abouzar, A. Poghossian, E. Katz, Field-effect nanoparticle-based glucose sensor on a chip: amplification effect of coimmobilized redox species, Electroanalysis 20 (2008) 1748-1753.

[21] M.H. Abouzar, A. Poghossian, A.G. Cherstvy, A.M. Pedraza, S. Ingebrandt, M.J Schöning, Label-free electrical detection of DNA by means of field-effect nanoplate capacitors: experiments and modeling, Physical Status Solidi A 209 (2012) 925-934

[22] K. Nakazato, An integrated ISFET sensor array, Sensors 9 (2009) 8831-8851.

[23] A. Poghossian, S. Ingebrandt, A. Offenhäusser, M. Schöning, Field-effect devices for detecting cellular signals, Seminars in Cell and Developmental Biology 20 (2009) 41-48.

[24] A. Demoz, E.M. Verpoorte, D.J. Harrison, An equivalent circuit model of ion-selective membrane|insulator|semiconductor interfaces used for chemical sensors, Journal of Electroanalytical Chemistry 389 (1995) 71-78.

[25] A. Poghossian, D.T. Mai, Y. Mourzina, M.J. Schöning Impedance effect of an ion-sensitive membrane: characterisation of an EMIS sensor by impedance spectroscopy, capacitance-voltage and constant-capacitance method, Sensors and Actuators B 103 (2004) 423-428.

[26] P. Fabry, L. Laurent-Yvonnou, The $C-V$ method for characterizing ISFET or EOS devices with ion-sensitive membranes, Journal of Electroanalytical Chemistry 286 (1990) 23-40.

[27] K.A. Athanasiou, G.G. Niederauer, C. Agrawal, Sterilization, toxicity, biocompatibility and clinical applications of polylactic acid/polyglycolic acid copolymers, Biomaterials 17 (1996) 93-102.

[28] G. Scott, Degradable Polymers: Principles and Applications, Second ed., Kluwer Academic Publishers, Dordrecht, The Netherlands, 2003.

[29] H. Tsuji, Hydrolytic degradation, Poly(Lactic Acid): Synthesis, Structures, Properties, Processing, and Applications, John Wiley \& Sons, Inc., Hoboken, New Jersey, USA, pp. 343-381.

[30] A. Poghossian, M.J. Schöning, Silicon-based chemical and biological field-effect sensors, Encyclopedia of Sensors, vol. 9, American Scientific Publisher, Santa Clarita, California, USA, pp. 463-534.

[31] A. Göpferich, Mechanisms of polymer degradation and erosion, Biomaterials 17 (1996) 103-114. 


\section{Biographies}

Sebastian Schusser received his diploma degree in physical engineering from Aachen University of Applied Sciences in 2009. Afterwards, he started his PhD thesis at the Institute of Nano- and Biotechnologies. Being a member of the Laboratory o Chemical Sensors and Biosensors at the Campus Jülich, his research subject focuse on (bio-)chemical sensors, especially capacitive field-effect sensors and impedance measurement.

Arshak Poghossian received his PhD degree in solid-state physics from Leningrad Electrotechnic Institute in 1978 and the Dr. Sci. degree in solid-state electronics and microelectronics from the State University of Yerevan (Armenia) in 1995. After being an associate professor of State Engineering University of Armenia and director of Microsensor Ltd. (Yerevan) from 1991 to 1996, he has been a professor at the University of Management and Information (Yerevan). Since 1998, he has been with the Institute of Thin Films and Interfaces (now, Peter Grünberg Institute-8) at the Research Centre Jülich, and since 2004, he joined the Institute of Nanoand Biotechnologies at Aachen University of Applied Sciences, Germany. In 2008 he has been appointed as Honorary Professor. He is a member of the Armenian Academy of Science. His research interests are solid-state chemical sensors and biosensors, sensor materials, nano-devices, microsystem technology, and nano- and biotechnology.

Marcel Leinhos received his diploma degree in bio- and nanotechnology from South Westphalia University of Applied Sciences in 2008. In 2009, he obtained his M.Sc. degree in electrical and information engineering from University Hagen. Since 2011 he is with the Institute of Nano- and Biotechnologies working on (bio-)chemical sensors for multisensor applications and the fabrication of sensors by silicon and thin-film technologies.

Matthias Bäcker received his diploma degree in biomedical engineering from Aachen University of Applied Sciences in 2007. Since then, he is with the Institute of Nano- and Biotechnologies working on chip-based chemical sensors and biosensors for fermentation monitoring and hydrogel sensors.

Patrick Wagner obtained his PhD in 1994 at Technical University Darmstadt (Germany) in experimental solid-state physics with a focus on cuprate superconductors. From 1995 until 2001, he was postdoctoral researcher in the Laboratory of Solid State Physics and Magnetism at Catholic University Leuven (Belgium), where he studied the magneto-transport properties of mixed-valency magnetic oxides. Since 2001, he is a professor of physics at Hasselt University (Belgium) and responsible for the development of label-free readout techniques for DNA-, protein-, and smallmolecule sensors. Patrick Wagner is recipient of a WE Heraeus award, a Marie-Curie Fellowship of the European Union, a Methusalem Grant of the Flemish Government and is past president of the Belgian Physical Society.

Michael J. Schöning received his PhD in 1993 at Karlsruhe University of Technology in the field of semiconductor-based microsensors for the detection of ions in liquids. From 1993 until 1999, he has been with the Institute of Thin Films and Interfaces (now, Peter Grünberg Institute-8) at the Research Center Jülich, and since 1999, he was appointed as full Professor at Aachen University of Applied Sciences, Campus Jülich. Since 2006, he serves as a director of the Institute of Nano- and Biotechnologies (INB) at the Aachen University of Applied Sciences. His main research subjects concern silicon-based chemical and biological sensors, thin-film technologies, solidstate physics, microsystem and nano(bio-)technology. 